Published in final edited form as:

Cancer Epidemiol. 2017 August ; 49: 186-194. doi:10.1016/j.canep.2017.07.001.

\title{
Survival after Recurrence of Stage I-III Breast, Colorectal, or Lung Cancer
}

\author{
Michael J. Hassett ${ }^{\mathrm{a}}$, Hajime Uno ${ }^{\mathrm{b}}$, Angel M. Cronin ${ }^{\mathrm{a}}$, Nikki M. Carroll ${ }^{\mathrm{c}}$, Mark C. Hornbrook ${ }^{\mathrm{d}}$, \\ Paul Fishman ${ }^{\mathrm{e}}$, and Debra P. Ritzwoller ${ }^{\mathrm{C}}$ \\ aDivision of Population Sciences, Dana-Farber Cancer Institute, Boston, MA \\ bHarvard Medical School, Boston, MA \\ 'Kaiser Permanente Colorado, Institute for Health Research, Denver, CO \\ ${ }^{\mathrm{d} K a i s e r}$ Permanente Center for Health Research, Portland, OR \\ eSchool of Public Health, University of Washington, Seattle, WA
}

\section{Abstract}

Background-The experiences of patients with recurrent cancer are assumed to reflect those of patients with de novo stage IV disease; yet, little is truly known because most registries lack recurrence status. Using two databases with excellent recurrence and death information, we examined determinants of survival duration after recurrence of breast (BC), colorectal (CRC), and lung cancers (LC).

Methods-Recurrence status was abstracted from the medical records of patients who participated in the Cancer Care Outcomes Research and Surveillance study and who received care at two Cancer Research Network sites - the Colorado and Northwest regions of Kaiser Permanente. The analysis included 1,653 patients who developed recurrence after completing definitive therapy for stages I-III cancer. Multivariable modeling identified independent determinants of survival duration after recurrence, controlling for other factors.

Results-Through 60 months' average follow-up, survival after recurrence for BC, CRC, and LC were 28.4, 23.1 and 16.1 months, respectively. Several factors were independently associated with shorter survival for all three cancers, including higher initial stage (III vs. I: BC -9.9 months; CRC -6.9 months; LC -7.4 months; $P \leq 01$ ). Factors associated with shorter survival for selected cancers included: distant/regional recurrence for $\mathrm{BC}$ and $\mathrm{CRC}$; current/former smoker for LC; high grade for CRC; and <4-year time-to-recurrence for BC.

Conclusions-Initial stage predicts survival duration after recurrence, whereas time-torecurrence usually does not. The impact of biologic characteristics (e.g., grade, hormone-receptor

Corresponding Author: Michael J. Hassett MD MPH, Dana-Farber Cancer Institute, 450 Brookline Avenue, Boston, MA 02215; PH: 617-632-6631; FX: 671-632-2270; michael_hassett@dfci.harvard.edu.

Publisher's Disclaimer: This is a PDF file of an unedited manuscript that has been accepted for publication. As a service to our customers we are providing this early version of the manuscript. The manuscript will undergo copyediting, typesetting, and review of the resulting proof before it is published in its final citable form. Please note that during the production process errors may be discovered which could affect the content, and all legal disclaimers that apply to the journal pertain. 
status) on survival duration after recurrence needs further study. Predictors of survival duration after recurrence may help facilitate patient decision-making.

\section{Keywords}

recurrence; survival; outcomes; natural history; breast cancer; lung cancer; colorectal cancer

\section{INTRODUCTION}

Nearly all cancer deaths occur as a result of metastatic disease. In some patients, metastatic disease is present when cancer is first diagnosed, but in many patients metastatic disease represents recurrence that develops among patients previously treated for earlier stage cancer. Most large data sets, including tumor registries, electronic medical records, and health insurance claims, do not capture recurrence status. Consequently, much of what we believe about metastatic cancer comes from patients with de novo stage IV disease. We face a dearth of population-based research on the patterns-of-care provided to and outcomes experienced by patients with recurrent cancer.

Among patients with newly diagnosed non-metastatic cancer, the factors that predict survival duration have been well described. Nevertheless, these same factors may not predict survival duration from the point of recurrence for the subset of patients whose cancers recur. While one may assume the experiences of patients with recurrent cancer mimic those of patients with de novo stage IV cancer, these experiences may differ for a number of reasons. Patients with recurrence may have more aggressive disease, considering that their cancer recurred despite prior therapy. Because of cumulative toxicity or dose limits, previously administered treatments may not be an option at the time of recurrence. And the treatment preferences of patients with recurrence could diverge from those with de novo stage IV disease.

Few reliable determinants of survival duration for recurrent or de novo metastatic cancer have been identified. Studies of locally recurrent breast cancer among patients initially treated on National Surgical Adjuvant Breast and Bowel Project (NSABP) clinical trials suggest that initial stage and time-to-recurrence influence outcomes.[1, 2] However, these studies did not include patients with distant recurrence, and little is known about outcomes after recurrence of other cancers. Investigators have looked at how treatments and surveillance practices impact development of recurrence and/or survival from the initial cancer diagnosis, but these studies usually did not focus on the events that followed recurrence.[3-8] Several National Comprehensive Cancer Network studies and many clinical trials have examined the association between patient features, tumor characteristics, and/or treatment factors, on survival among patients with metastatic cancer. These analyses, however, usually grouped patients with recurrent and stage IV disease together.[9, 10], and the treatments provided to and outcomes experienced by patients treated in these research settings may not reflect those of patients treated in community settings.

Our goals were to use a unique pair of datasets that offered high-quality information on cancer incidence, recurrence, and health services utilization to identify factors associated with survival duration following recurrence of breast, colorectal, and lung cancers. We also 
described patterns-of-care among patients with recurrent cancer treated in community settings.

\section{MATERIALS and METHODS}

\subsection{Data Sources}

The databases used in this analysis came from the Cancer Care Outcomes Research and Surveillance (CanCORS) Consortium, and the Cancer Research Network (CRN). CanCORS was a large, prospective, population and health-system based study of lung and colorectal cancer patients diagnosed in 2003-2005.[11, 12] Data collected through CanCORS were derived from multiple data sources through extensive medical records reviews and patient surveys. The CRN (http://crn.cancer.gov/) is a consortium of large health care systems affiliated with the Health Care Systems Research Network (HCSRN) and the National Cancer Institute. Two CRN sites, whose certified tumor registrars collect high quality recurrence data, contributed to this analysis: Kaiser Permanente Colorado, Denver, CO, and Kaiser Permanente Northwest, Portland, OR. The CRN maintains a Virtual Data Warehouse (VDW)[13] that links tumor registry data, diagnosis and procedure codes documented in an EPIC ${ }^{\circledR}$-based EHR, claims for services delivered by external contract providers, health plan eligibility, and member demographic data.

Institutional Review Boards from the Dana-Farber/Harvard Cancer Center and the participating CRN sites approved and provided oversight for the project.

\subsection{Study Cohorts and Recurrence Status}

All patients 1) were diagnosed with invasive, non-metastatic breast (BC), colorectal (CRC), or lung (LC) cancer when $\geq 21$ years old; 2) completed definitive local-regional therapy for their initial cancer; 3 ) survived and were followed for at least 30 days after definitive therapy; and, 4) subsequently experienced recurrence. Definitive local-regional therapy was defined as organ-directed surgery appropriate for the specific cancer (i.e., mastectomy for $\mathrm{BC}$, colectomy for CRC, lobectomy for LC), with or without radiation therapy. For patients with stage IIIa lung cancer, receiving both chemotherapy and radiation therapy was also considered definitive local-regional therapy. Patients with stage IV BC and CRC, and stages IIIb-IV LC were excluded, as were those who developed recurrence or died before definitive therapy or had another cancer diagnosis (Figure 1).

Presence of recurrence and date of recurrence were derived from the abstracted medical record and/or tumor registry.[13, 14] Recurrent events were classified as "local only" or "regional/distant". Unless explicity stated, 'recurrence' herein refers to both types of events (i.e., local and regional/distant). Vital status was ascertained from health plan records or state and national death records. CanCORS patients were followed until death or study end date (12/31/11). CRN patients were followed through death, health plan disenrollment, or study end date $(12 / 31 / 12)$.

Patient and disease characteristics were derived from survey and/or medical record abstraction for CanCORS and from tumor registry data for CRN patients. Notably, income was collected at the individual level for CanCORS and at the census block level for the 
CRN. A co-morbidity score was dervied using established methods[15-17], excluding cancer diagnoses. Chemotherapy and radiation therapy treatments within 12 months following the initial diagnosis and within 3 months following the recurrence were identified using medical record data for CanCORS, and EHR procedure, pharmacy, and infusion files for the CRN.[18]

\subsection{Statistical Methods}

First, we conducted a number of exploratory analyses to determine whether or not it was appropriate to identify determinants for the pooled sample (i.e., CRN and CanCORS together), rather than for the two datasets separately. As noted above, there were several differences between the CanCORS and CRN data. First, CanCORS patients consented to participate in a research study, whereas CRN patients received care at participating sites. Second, CanCORS studied colorectal and lung cancers diagnosed 2003-2005, whereas the CRN included colorectal, lung, and breast cancers diagnosed 2000-2011. Third, CanCORS data were derived from manual medical record abstraction, whereas CRN data came mainly from the electronic health records (EHR) of an integrated managed care consortium. We found no significant differences for LC and CRC patients who received care in CanCORS relative to those who received care in the CRN. Moreover, preliminary analyses conducted separately by data source largely identified similar predictors of survival duration after recurrence. In the models (described below) we added interaction terms between each covariate and the data source and confirmed that there was no evidence of heterogeneity between models containing versus models excluding these interaction terms. Since no significant interactions between the data source and predictors were observed, we pooled the samples and fit one multivariable model for each cancer type while controlling for data source.

Restricted mean survival time (RMST) was chosen as the primary outcome because it does not rely on assumptions regarding proportional hazards, and it generates clinically-relevant effect estimates. For example, the RMST reports the mean number of months of survival gained or lost when a relevant characteristic is present, whereas the Cox model produces a hazard ratio which would have a different interpretation if the contorl population had an average survival of 4 months compared to 24 months. The use of RMST as a primary outcome has been adovacted by methodologists and is increasingly being used in cancer clinical investigations.[19] Multivariable models predicted RMST through 60 months, to focus on a clinically relevant time-point and facilitate comparisions between cancers. All variables of interest (see Table 1) were included regardless of statistical significance. Two predictors (chemotherapy and radiotherapy for recurrence) were subject to guaranteed time bias, so models were restricted to patients who survived beyond the three-month landmark time-point (the period during which these treatment were assessed). We did not intend to conduct a formal comparative effectiveness study of treatments for recurrence, but rather to adjust estimates of other predictors for treatments received and to explore estimates of the treatment variables. Median survival and two-year survival probabilities were also reported to facilitate comparability with other published literature. 
Patients were missing values as follows: income (12\%); race/ethnicity (7\%); tumor grade (4\%); marital status (9\%); smoking status (9\%); and hormone receptor (HR) status (43\% of the $\mathrm{BC}$ cohort). Multiple imputation by chained equations were used to impute missing values separately by data source and cancer type. All statistical analyses were conducted using Stata version 13.1 (StataCorp LP, College Station, TX). $P$ values $<.05$ were considered significant.

\section{RESULTS}

The proportions of patients who developed recurrence after being treated for stage I-III BC, CRC, and LC were 7\%, 13\%, and 27\%, respectively, through median follow-ups of 57, 35, and 25 months. Recurrent BC patients, all from the CRN, were relatively evenly distributed across age groups (Table 1). Over half resided in neighborhoods with average annual household incomes $>\$ 60,000$. The majority had low comorbidity burden (modified Charlson-Deyo index $=0$ ). Receipt of chemotherapy and radiotherapy were more common after the primary cancer ( $71 \%$ and $67 \%$, respectively) than the recurrence $(58 \%$ and $31 \%$, respectively). Approximately four-fifths ( $81 \%$ ) of recurrences were regional/distant.

We identified $670 \mathrm{CRC}$ and $478 \mathrm{LC}$ recurrent patients from the CRN (54\%) and CanCORS (46\%). Both cohorts were heavily distributed in the older age categories, with $46 \%$ of CRC and $47 \%$ of LC age $>70$ years. Relative to BC patients, the income categories for $\mathrm{LC}$ and CRC patients were more evenly distributed. The comorbidity burden was highest in LC ( $\geq 2$ for 33\%), as was the likelihood of being a current/former smoker (80\%). Receipt of chemotherapy and radiotherapy for the primary cancer was $66 \%$ and $24 \%$, respectively for CRC; and $41 \%$ and $25 \%$, respectively for LC. The vast majority of CRC and LC recurrences were distant (93\%). Receipt of chemotherapy and radiation therapy following recurrence was $40 \%$ and $7 \%$ for CRC, and $33 \%$ and $26 \%$ for LC.

Among the subset of recurrent patients who died during the follow up-period, those with LC had the shortest median time from diagnosis to recurrence (14 months), followed by CRC (16 months), and BC (28 months). LC patients also had the shortest median time from recurrence to death (7 months), compared with CRC (12 months), and BC (11 months) patients. To facilitate comparability with other studies, estimates of survival duration following recurrence are reported as RMST through 60 months, median survival, and 2-year survival (Table 2). The RMST through 60 months was 28.4 months for BC, 23.1 months for CRC, and 16.1 months for LC. Higher unadjusted RMST was noted for BC patients with HR positive disease and greater time to recurrence, and for LC patients with higher incomes and those who never smoked. RMST was inversely related to the comorbidity score, stage at diagnosis, and grade at diagnosis for all cancers. Kaplan-Meier plots depict survival from recurrence stratified by type of recurrence, stage of primary, and time from primary to recurrence (Figure 2).

Multivariable models identified three factors independently associated with inferior RMST after recurrence for all three cancers (Table 3). The coefficient value listed for each characteristic represents the number of additional months of survival gained or lost if that characteristic was present, relative to the reference group for that covariate. Older patients 
had shorter RMST, with the effect being most pronounced in CRC ( -0.3 months/year; $P<$. $01)$. Patients with a higher comorbidity burden ( $2+$ vs. 0$)$ experienced inferior outcomes, with the largest decline in $\mathrm{BC}(-10.0$ months; $P=0.01)$. Patients initially diagnosed with stage III (vs. stage I) cancer had shorter RMST, with reductions of 10.3 months in BC, 6.8 months in CRC, and 7.5 months in LC ( $P \leq 0.02$ for each cancer).

A number of factors were independently associated with RMST in some, but not all cancers. Having distant/regional (vs. local) recurrence had the largest negative effect on RMST, but it only impacted patients with BC ( -19.1 months; $P<.01)$ and CRC ( -23.0 months; $P<.01)$. Longer time from primary cancer to recurrence was not significantly associated with longer RMST for any cancer, though a borderline result of longer survival was observed among BC patients when the time interval was $>4$ years ( +8.8 months; $P=0.06$ ). Having high (vs. low/ intermediate) grade cancer was associated with inferior RMST after recurrence for CRC ( -5.7 months; $P<.01$ ), but not BC or LC. Neither chemotherapy nor radiation therapy was significantly associated with RMST, whether given after the primary diagnosis or the recurrence, except that radiation after CRC recurrence was associated with shorter RMST $(-9.5$ months; $P<.01)$.

We conducted several sensitivity analyses (data not shown). Models excluding predictors subject to guaranteed time bias (i.e., chemotherapy and radiation after recurrence), and models predicting RMST through maximal follow-up (84 months for BC, 96 months for $\mathrm{CRC}$, and 72 months for $\mathrm{LC}$ ) demonstrated similar results. Also, restricting the $\mathrm{BC}$ cohort to patients diagnosed after 2003 - to exclude patients with unknown HR status-found no significant association between HR status and survival duration after recurrence.

\section{CONCLUSIONS}

To better understand the treatments provided to and outcomes experienced by patients with recurrent cancer, we studied 1,653 patients who developed recurrence after having had treatment for non-metastatic $\mathrm{BC}, \mathrm{CRC}$, or $\mathrm{LC}$. The median time from primary cancer to recurrence was approximately twice as long for $\mathrm{BC}$ patients compared to those with CRC and $\mathrm{LC}$. The median overall survival after recurrence was longest for $\mathrm{BC}$ and $\mathrm{CRC}$, followed by LC patients. Local recurrences were uncommon, especially for CRC and LC. Notably, $\mathrm{BC}$ and CRC, but not LC patients, experienced better survival after local versus regional/ distant recurrence. Initial stage was the only pre-recurrence characteristic significantly associated with survival after recurrence for all three cancers. While previous studies have demonstrated that stage is negatively associated with survival following the initial diagnosis, we are not aware of studies showing that stage predicts survival after the development of recurrence across cancer types. Only $\mathrm{BC}$ patients experienced better outcomes when time-torecurrence was longer, and this benefit was only seen among patients with a time to recurrence $>4$ years.

Grade, a generally accepted prognostic factor for newly-diagnosed cancer patients, was only associated with longer survival after recurrence for CRC, but not BC or LC patients. Unexpectedly, HR status was not associated with longer survival after BC recurrence when controlling for other factors. Our sample sizes may have been insufficient to detect a 
significant benefit for HR status. Alternatively, the survival benefit associated with HR positivity may be less substantial for patients with recurrent versus de novo metastatic breast cancer, perhaps because recurrent patients previously received anti-estrogen therapy. These findings help address the need for reliable determinants of survival for patients with recurrent cancer. Still, additional studies should explore the extent to which tumor biology, extent of disease, host-tumor interactions, or other factors may help predict survival after recurrence.

Chemotherapy after recurrence was less common than chemotherapy after the primary cancer diagnosis, and most common for patients with BC (BC 58\%, CRC 40\%, and LC $33 \%$ ). Radiation after recurrence was observed in approximately one-quarter of $\mathrm{BC}$ and $\mathrm{LC}$, but only $7 \%$ of CRC patients. Few studies have assessed the care provided to or outcomes experienced by patients with recurrent disease, but a number have used SEER data to study patients with de novo stage IV metastatic cancer. For example, among patients with stage IV BC, use of chemotherapy was seen in 52\%, two-year overall survival was 40-46\%, and median survial was 23 months.[20, 21] In metastatic CRC, $41 \%$ received chemotherapy, and the 2 year survival rate was approximately $22 \%$.[22-24] For stage IV lung cancer, approximately $30 \%$ got chemotherapy, and the 2 year survival rate was $<10 \%$.[25-28] Observing that the patterns of care from our study are similar to the patterns of care for patients with de novo stage IV cancer described by prior studies suggests that receipt of prior therapy had little impact on treatment for recurrence. However, we were not able to test this hypothesis directly, because our cohort only included patients with recurrent metastatic cancer. In light of observations that clinical trial results for patients with advanced cancer may overestimate outcomes observed in SEER, [29] future studies should assess the extent to which treatment effectiveness and survival differ for patients with recurrent versus stage IV cancer.

Our analysis was not designed to compare the effectiveness of different treatment strategies for patients with recurrent cancer. However, exploratory analyses demonstrated that neither systemic therapy for the initial diagnosis nor systemic therapy for the recurrence was associated with inferior survival after recurrence. These findings reinforcing the long-held belief that the primary goal of chemotherapy for recurrence is palliation. They also suggest that systemic therapy for an initial diagnosis does not impact survival duration from the point at which recurrent disease develops. That said, chemotherapy for an initial nonmetastatic diagnosis could still prolong survival among patients who eventually experience a recurrence by delaying the time until recurrence develops.

While the methods used to determine who had recurrence were exhaustive, some recurrences could have been missed. Some important covariates lacked complete data (e.g., HR status for BC), potentially limiting the power to detect an association between HR status and survival after recurrnece. The CRN was the only source of data for patients with recurrent $\mathrm{BC}$; reassuringly, analyses of CRC and LC patients found no meaningful differences in outcomes between the CanCORS and CRN sampels. Our analysis focused on treatments administered within 3 months of recurrence, so there could have been under-reporting or censoring of treatments for recurrence after 3 months. Some data collection methods differed (e.g., smoking was collected from a patient survey in CanCORs and from the 
medical record in the $\mathrm{CRN}$ ), but we believe these differences bear little weight on the primary findings. Lastly, our sample only included patients who had enrolled in CanCORS or received care in the capitation-based Kaiser Permanente network.

Comparing data for cancer incidence and cancer mortality, the number of patients who die from cancer far exceeds the number diagnosed with stage IV disease.[30, 31] Cancer deaths not caused by stage IV disease must be attributable to recurrence. For some cancers, such as $\mathrm{BC}$, the death rate is falling as the incidence of stage IV disease remains steady, [31, 32] suggesting that the incidence of recurrent disease is falling. If so, then the nature of recurrent cancer, including the extent to which it behaves like de novo stage IV disease, may be changing over time. We have tried to fill in some gaps regarding recurrent cancer, but important questions remain. If substantial differences between recurrent and stage IV cancer do exist, they could impact prognostic estimates, treatment choices, and the way clinical trials are designed. We recommend that population-based tumor registries devote resources to identifying patients who develop recurrence to facilitate future analyses of this clinically important population.

\section{Acknowledgments}

Funding: This work was supported by a grant from the National Cancer Institute (R01 CA172143 to MJH/DPR) and an NCI Cooperative Agreement (U19 CA79689 to the Cancer Research Network). The American Society of Clinical Oncology (Career Development Award) and Susan G. Komen for the Cure (Career Catalyst Award) provided salary support to MJH. The work of the CanCORS consortium was supported by grants from the National Cancer Institute (NCI) to the Statistical Coordinating Center (U01 CA093344) and the NCI supported Primary Data Collection and Research Centers (Dana-Farber Cancer Institute/Cancer Research Network U01 CA093332).

\section{References}

1. Anderson SJ, Wapnir I, Dignam JJ, Fisher B, Mamounas EP, Jeong JH, Geyer CE Jr, Wickerham DL, Costantino JP, Wolmark N. Prognosis after ipsilateral breast tumor recurrence and locoregional recurrences in patients treated by breast-conserving therapy in five National Surgical Adjuvant Breast and Bowel Project protocols of node-negative breast cancer. Journal of clinical oncology: official journal of the American Society of Clinical Oncology. 2009; 27(15):2466-2473. [PubMed: 19349544]

2. Wapnir IL, Anderson SJ, Mamounas EP, Geyer CE Jr, Jeong JH, Tan-Chiu E, Fisher B, Wolmark N. Prognosis after ipsilateral breast tumor recurrence and locoregional recurrences in five National Surgical Adjuvant Breast and Bowel Project node-positive adjuvant breast cancer trials. Journal of Clinical Oncology. 2006; 24(13):2028-2037. [PubMed: 16648502]

3. Lee HY, Lee SW, Lee KS, Jeong JY, Choi JY, Kwon OJ, Song SH, Kim EY, Kim J, Shim YM. Role of CT and PET Imaging in Predicting Tumor Recurrence and Survival in Patients with Lung Adenocarcinoma: A Comparison with the International Association for the Study of Lung Cancer/ American Thoracic Society/European Respiratory Society Classification of Lung Adenocarcinoma. J Thorac Oncol. 2015

4. Chen P, Yao Y, Zhao J, Li M, Peng Y, Zhan T, Du C, Wang L, Chen N, Gu J. Retrospective analysis of 856 cases with stage 0 to III rectal cancer underwent curative surgery combined modality therapy. Zhonghua wai ke za zhi [Chinese journal of surgery]. 2015; 53(7):496-501.

5. Park S, Han W, Kim J, Kim MK, Lee E, Yoo TK, Lee HB, Kang YJ, Kim YG, Moon HG, et al. Risk Factors Associated with Distant Metastasis and Survival Outcomes in Breast Cancer Patients with Locoregional Recurrence. J Breast Cancer. 2015; 18(2):160-166. [PubMed: 26155292]

6. Adams K, Higgins L, Beazley S, Papagrigoriadis S. Intensive surveillance following curative treatment of colorectal cancer allows effective treatment of recurrence even if limited to 4 years. International journal of colorectal disease. 2015; 30(12):1677-1684. [PubMed: 26320020] 
7. Lewis C, Xun P, He K. Effects of adjuvant chemotherapy on recurrence, survival, and quality of life in stage II colon cancer patients: a 24-month follow-up. Supportive care in cancer: official journal of the Multinational Association of Supportive Care in Cancer. 2015

8. MC, XL, AMG-A, EAM, VV, DT, Hortobagyi G, MC-M. Recurrence and survival among breast cancer patients achieving a pathological complete response to neoadjuvant chemotherapy. Breast cancer research and treatment. 2015; 153(2):417-423. [PubMed: 26272743]

9. Matro JM, Li T, Cristofanilli M, Hughes ME, Ottesen RA, Weeks JC, Wong YN. Inflammatory breast cancer management in the national comprehensive cancer network: the disease, recurrence pattern, and outcome. Clinical breast cancer. 2015; 15(1):1-7. [PubMed: 25034439]

10. Warner ET, Tamimi RM, Hughes ME, Ottesen RA, Wong YN, Edge SB, Theriault RL, Blayney DW, Niland JC, Winer EP, et al. Racial and Ethnic Differences in Breast Cancer Survival: Mediating Effect of Tumor Characteristics and Sociodemographic and Treatment Factors. Journal of clinical oncology: official journal of the American Society of Clinical Oncology. 2015; 33(20): 2254-2261. [PubMed: 25964252]

11. Ayanian JZ, Chrischilles EA, Fletcher RH, Fouad MN, Harrington DP, Kahn KL, Kiefe CI, Lipscomb J, Malin JL, Potosky AL, et al. Understanding cancer treatment and outcomes: the Cancer Care Outcomes Research and Surveillance Consortium.[see comment][erratum appears in J Clin Oncol. 2004 Dec 15;22(24):5026]. Journal of clinical oncology: official journal of the American Society of Clinical Oncology. 2004; 22(15):2992-2996. [PubMed: 15284250]

12. Catalano PJ, Ayanian JZ, Weeks JC, Kahn KL, Landrum MB, Zaslavsky AM, Lee J, Pendergast J, Harrington DP. Cancer Care Outcomes Research Surveillance C. Representativeness of participants in the cancer care outcomes research and surveillance consortium relative to the surveillance, epidemiology, and end results program. Medical care. 2013; 51(2):e9-15. [PubMed: 22406968]

13. Hassett MJ, Ritzwoller DP, Taback N, Carroll N, Cronin AM, Ting GV, Schrag D, Warren JL, Hornbrook MC, Weeks JC. Validating Billing/Encounter Codes as Indicators of Lung, Colorectal, Breast, and Prostate Cancer Recurrence Using 2 Large Contemporary Cohorts. Medical care. 2012

14. Ritzwoller DP, Carroll N, Delate T, O’Keeffe-Rossetti M, Fishman PA, Loggers ET, Aiello Bowles EJ, Elston-Lafata J, Hornbrook MC. Validation of electronic data on chemotherapy and hormone therapy use in HMOs. Medical care. 2013; 51(10):e67-73. [PubMed: 22531648]

15. Katz JN, Chang LC, Sangha O, Fossel AH, Bates DW. Can comorbidity be measured by questionnaire rather than medical record review? Medical care. 1996; 34(1):73-84. [PubMed: 8551813]

16. Klabunde CN, Potosky AL, Legler JM, Warren JL. Development of a comorbidity index using physician claims data. Journal of Clinical Epidemiology. 2000; 53(12):1258-1267. [PubMed: 11146273]

17. Charlson ME, Pompei P, Ales KL, MacKenzie CR. A new method of classifying prognostic comorbidity in longitudinal studies: development and validation. Journal of Chronic Diseases. 1987; 40(5):373-383. [PubMed: 3558716]

18. Ritzwoller DP, Carroll N, Delate T, O’Keeffe-Rossetti M, Fishman PA, Loggers ET, Aiello Bowles EJ, Elston Lafata J, Hornbrook M. Validation of electronic data on chemotherapy and hormone therapy use in HMOs. Medical care. 2013; 51(10):e67-73. [PubMed: 22531648]

19. Trinquart L, Jacot J, Conner SC, Porcher R. Comparison of Treatment Effects Measured by the Hazard Ratio and by the Ratio of Restricted Mean Survival Times in Oncology Randomized Controlled Trials. Journal of clinical oncology: official journal of the American Society of Clinical Oncology. 2016

20. Dawood S, Broglio K, Gonzalez-Angulo AM, Buzdar AU, Hortobagyi GN, Giordano SH. Trends in survival over the past two decades among white and black patients with newly diagnosed stage IV breast cancer. Journal of clinical oncology: official journal of the American Society of Clinical Oncology. 2008; 26(30):4891-4898. [PubMed: 18725649]

21. Thomas A, Khan SA, Chrischilles EA, Schroeder MC. Initial Surgery and Survival in Stage IV Breast Cancer in the United States, 1988-2011. JAMA surgery. 2015:1-8.

22. Golan T, Urban D, Berger R, Lawrence YR. Changing prognosis of metastatic colorectal adenocarcinoma: Differential improvement by age and tumor location. Cancer. 2013; 119(16): 3084-3091. [PubMed: 23720150] 
23. Meyerhardt JA, Li L, Sanoff HK, Carpenter Wt, Schrag D. Effectiveness of bevacizumab with firstline combination chemotherapy for Medicare patients with stage IV colorectal cancer. Journal of clinical oncology: official journal of the American Society of Clinical Oncology. 2012; 30(6):608615. [PubMed: 22253466]

24. Bikov KA, Mullins CD, Seal B, Onukwugha E, Hanna N. Algorithm for identifying chemotherapy/ biological regimens for metastatic colon cancer in SEER-Medicare. Medical care. 2015; 53(8):e58-64. [PubMed: 23552436]

25. Laccetti AL, Pruitt SL, Xuan L, Halm EA, Gerber DE. Effect of prior cancer on outcomes in advanced lung cancer: implications for clinical trial eligibility and accrual. Journal of the National Cancer Institute. 2015; 107(4)

26. Karve SJ, Price GL, Davis KL, Pohl GM, Smyth EN, Bowman L. Comparison of demographics, treatment patterns, health care utilization, and costs among elderly patients with extensive-stage small cell and metastatic non-small cell lung cancers. BMC health services research. 2014; 14:555. [PubMed: 25392276]

27. Owonikoko TK, Ragin C, Chen Z, Kim S, Behera M, Brandes JC, Saba NF, Pentz R, Ramalingam SS, Khuri FR. Real-world effectiveness of systemic agents approved for advanced non-small cell lung cancer: a SEER-Medicare analysis. The oncologist. 2013; 18(5):600-610. [PubMed: 23635558]

28. Cetin K, Ettinger DS, Hei YJ, O’Malley CD. Survival by histologic subtype in stage IV nonsmall cell lung cancer based on data from the Surveillance, Epidemiology and End Results Program. Clinical epidemiology. 2011; 3:139-148. [PubMed: 21607015]

29. Lamont EB, Schilsky RL, He Y, Muss H, Cohen HJ, Hurria A, Meilleur A, Kindler HL, Venook A, Lilenbaum R, et al. Generalizability of trial results to elderly Medicare patients with advanced solid tumors (Alliance 70802). Journal of the National Cancer Institute. 2015; 107(1):336. [PubMed: 25432408]

30. Society AC. Cancer Facts \& Figures 015. Atlanta, GA: American Cancer Society; 2015.

31. Program, SR. Fast Stats: An interactive tool for access to SEER cancer statistics. National Cancer Institute; 2015.

32. Welch HG, Gorski DH, Albertsen PC. Trends in Metastatic Breast and Prostate Cancer--Lessons in Cancer Dynamics. New England journal of medicine. 2015; 373(18):1685-1687. [PubMed: 26510017]

\section{Authorship Contribution Statement}

\begin{tabular}{|l|l|l|l|l|l|l|l|l|}
\hline & $\begin{array}{l}\text { Concepti } \\
\text { on and } \\
\text { design }\end{array}$ & $\begin{array}{l}\text { Collecti } \\
\text { on and } \\
\text { assembl } \\
\text { y of } \\
\text { data }\end{array}$ & $\begin{array}{l}\text { Dana } \\
\text { analysis } \\
\text { and } \\
\text { interpretati } \\
\text { on }\end{array}$ & $\begin{array}{l}\text { Manuscri } \\
\text { pt writing }\end{array}$ & $\begin{array}{l}\text { Final } \\
\text { approval } \\
\text { of } \\
\text { manuscri } \\
\text { pt }\end{array}$ & $\begin{array}{l}\text { Financi } \\
\text { al } \\
\text { support }\end{array}$ & $\begin{array}{l}\text { Administrati } \\
\text { ve support }\end{array}$ & $\begin{array}{l}\text { Provisi } \\
\text { on of } \\
\text { study } \\
\text { materia } \\
\text { ls or } \\
\text { patients }\end{array}$ \\
\hline Michael Hassett & $\mathrm{X}$ & $\mathrm{X}$ & $\mathrm{X}$ & $\mathrm{X}$ & $\mathrm{X}$ & $\mathrm{X}$ & $\mathrm{X}$ & $\mathrm{X}$ \\
\hline Hajime Uno & $\mathrm{X}$ & & $\mathrm{X}$ & $\mathrm{X}$ & $\mathrm{X}$ & & & \\
\hline Angel Cronin & & & $\mathrm{X}$ & $\mathrm{X}$ & $\mathrm{X}$ & & & \\
\hline Nikki Carroll & & $\mathrm{X}$ & $\mathrm{X}$ & $\mathrm{X}$ & $\mathrm{X}$ & & & \\
\hline Mark Hornbrook & $\mathrm{X}$ & $\mathrm{X}$ & $\mathrm{X}$ & $\mathrm{X}$ & $\mathrm{X}$ & & $\mathrm{X}$ & $\mathrm{X}$ \\
\hline Paul Fishman & & & $\mathrm{X}$ & $\mathrm{X}$ & $\mathrm{X}$ & & & $\mathrm{X}$ \\
\hline Debra Ritzwoller & $\mathrm{X}$ & $\mathrm{X}$ & $\mathrm{X}$ & $\mathrm{X}$ & $\mathrm{X}$ & $\mathrm{X}$ & & $\mathrm{X}$ \\
\hline
\end{tabular}




\section{Highlights}

- Initial stage predicts survival after cancer recurrence

- Time-to-recurrence is not a consistent determinant of survival after recurrence

- Predictors of survival after recurrence may help facilitate patient decisionmaking 


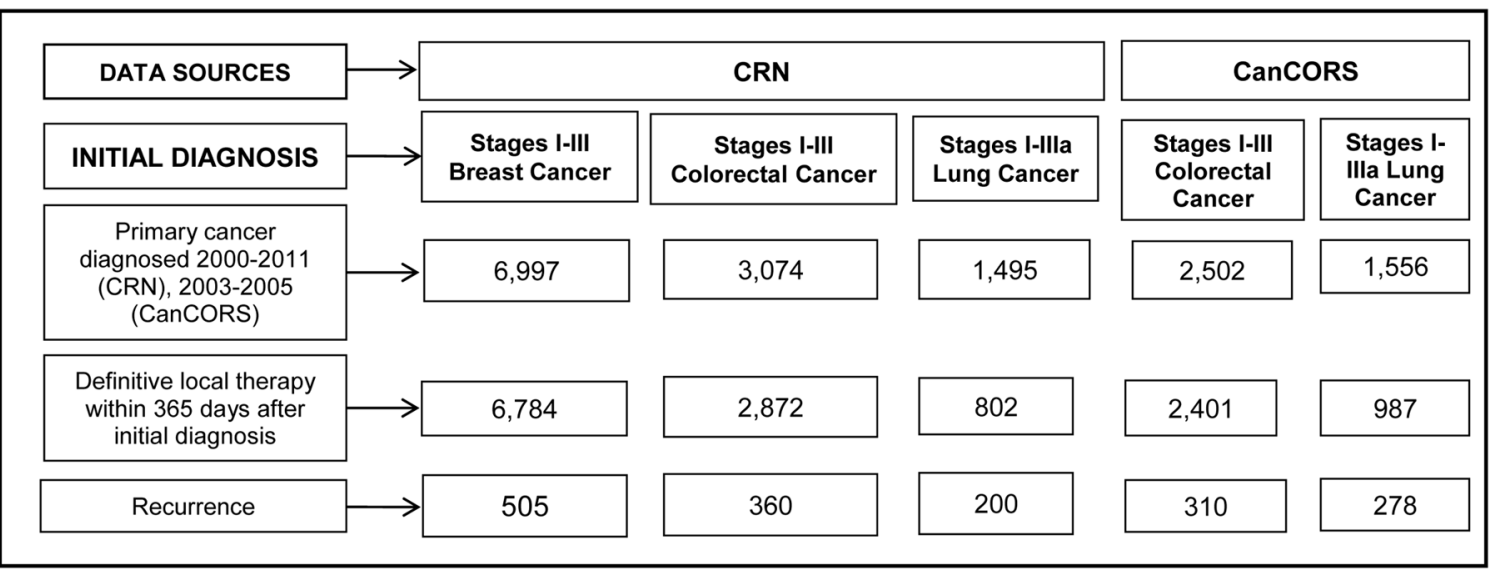

Figure 1. Flow diagram of breast, colorectal and lung cancer patients from the CRN and CanCORS who were included in this analysis

For the Cancer Research Network (CRN) cohort, inclusion criteria included: first cancer diagnosis only (i.e., no previous cancer diagnosis), enrolled in one of two participating CRN institutions at diagnosis, and at least age 21 at diagnosis. For the Cancer Care Outcomes Research and Surveillance Consortium (CanCORS) cohort, inclusion criteria included: CanCORS participant, first cancer diagnosis only (i.e., no previous cancer diagnosis), at least age 21 at diagnosis, and both baseline interview and medical record abstraction data were available. CanCORS only enrolled colorectal and lung cancer patients so did not contribute breast cancer patients to this analysis. Patients from Kaiser Permanente Northwest who enrolled in CanCORS were excluded from the CanCORS cohort, because they were part of the CRN cohort. All patients must have had stage I-III cancer (excluding stage IIIb lung cancer) treated with definitive local therapy within 365 days after the initial cancer diagnosis. Also, patients must have been followed for $>1$ day after recurrence (only one patient, from the $\mathrm{CRN}$ with colorectal cancer, was excluded due to lack of follow-up after recurrence). 

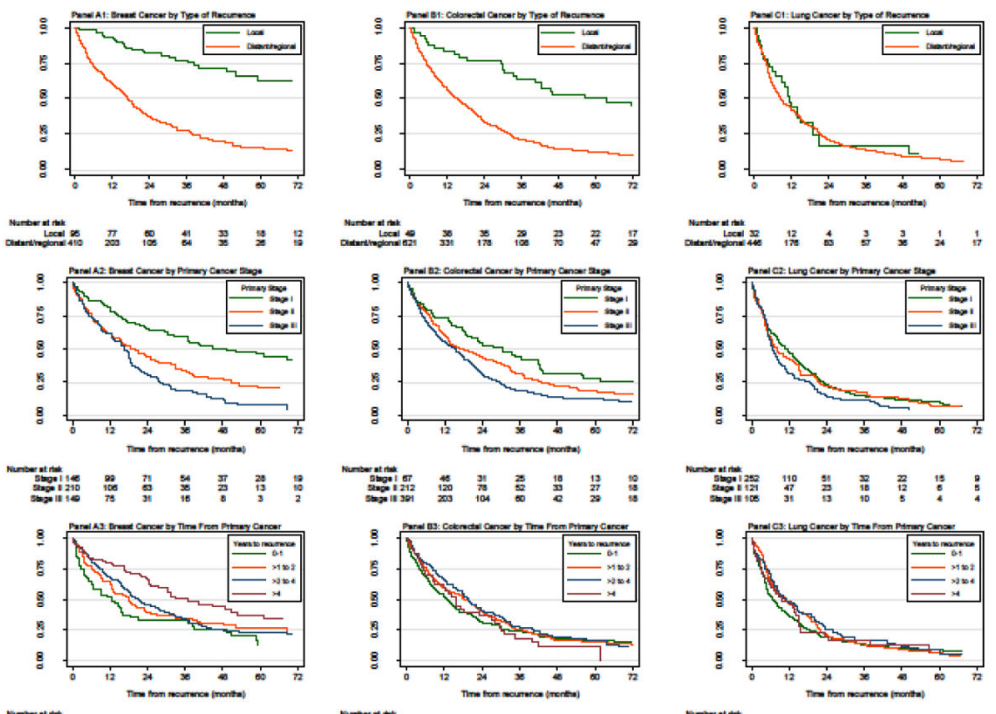

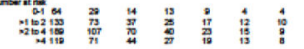
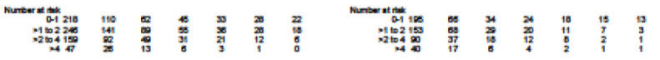

Figure 2. Kaplan-Meier plots of overall survival following recurrence through 60 months of follow-up for patients with breast (panel A), colorectal (panel B), and lung (panel C) cancer Outcomes were stratified by type of recurrence (local vs. distant/regional) in panels A1-C1; stage at initial cancer diagnosis (I, II, and III) in panels A2-C2; and time from initial cancer diagnosis to recurrence ( 0 to $1,>1$ to $2,>2$ to 4 , and $>4$ years) in panels A3-C3. 


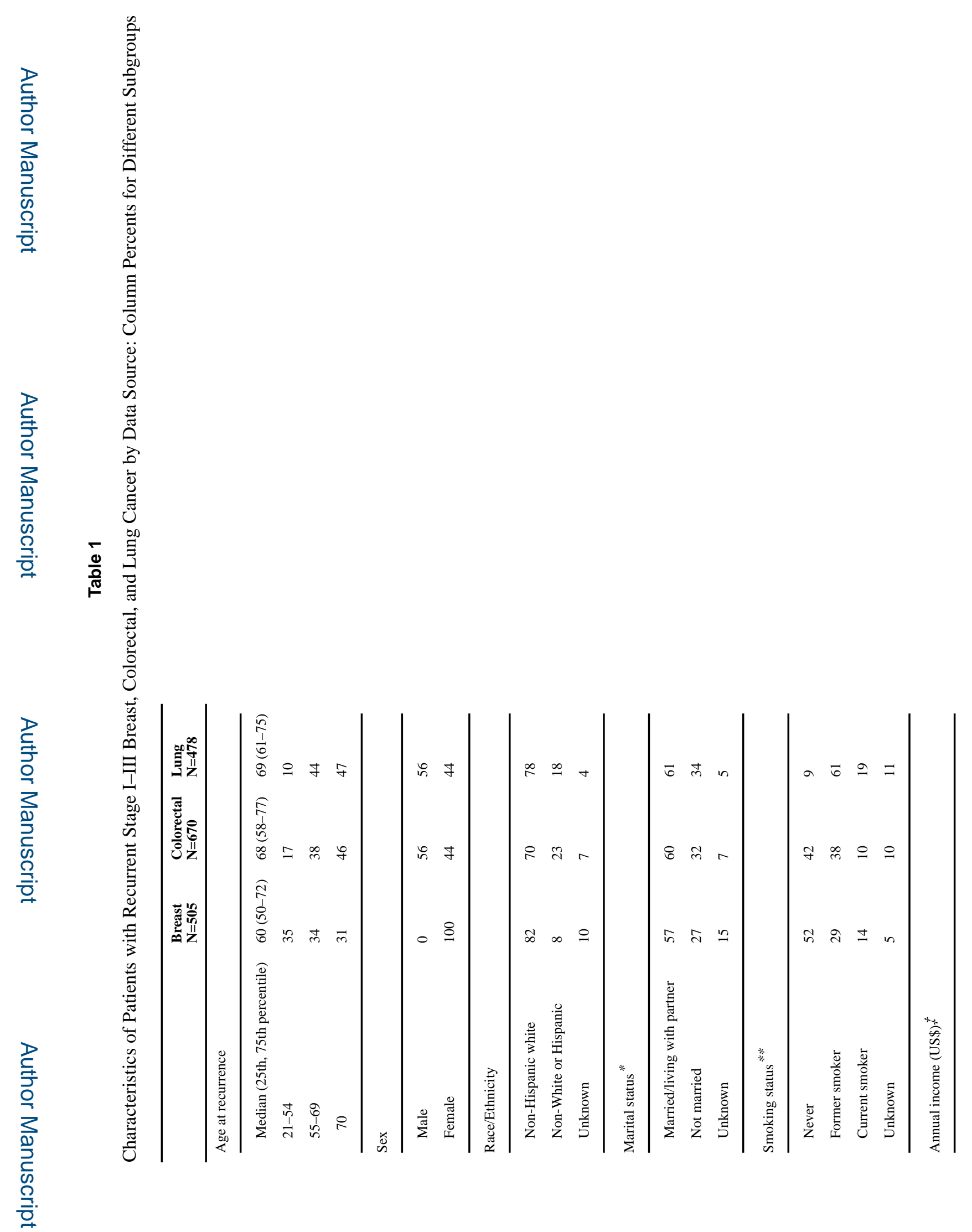


Hassett et al.

Page 15

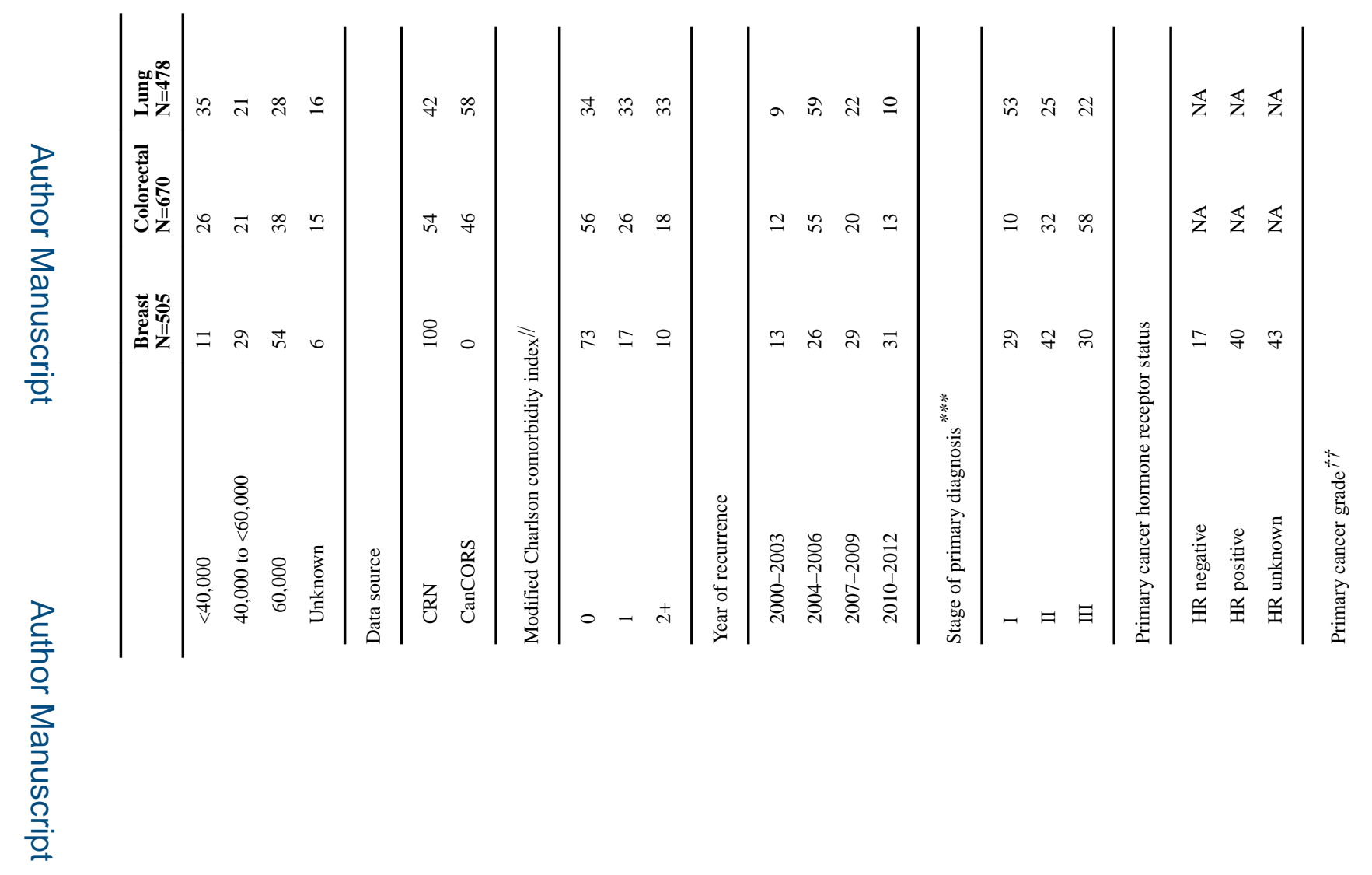

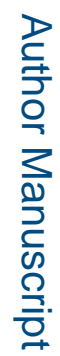

를

Cancer Epidemiol. Author manuscript; available in PMC 2018 August 01. 


$$
\text { 䍩 }
$$

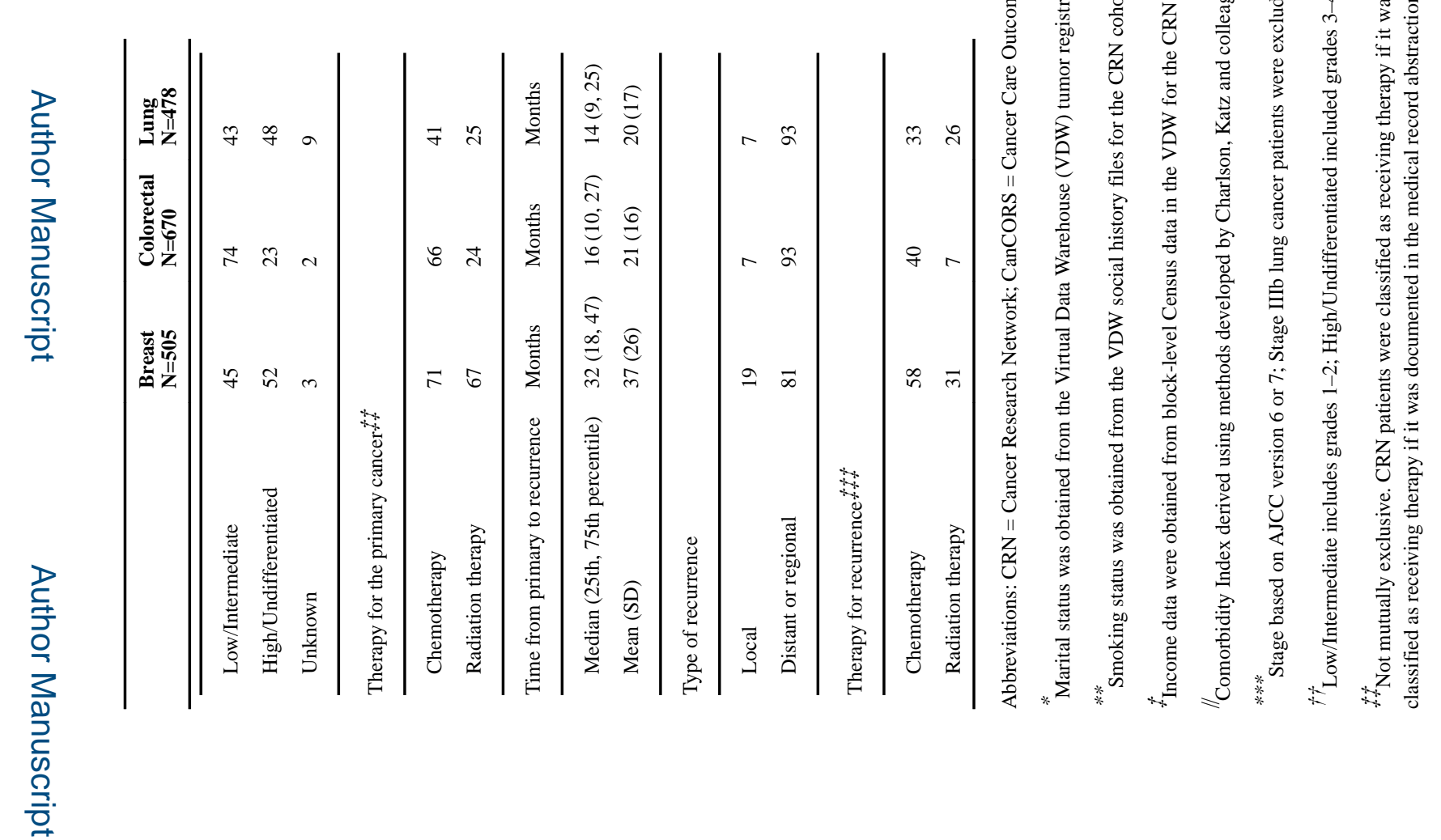

Cancer Epidemiol. Author manuscript; available in PMC 2018 August 01. 


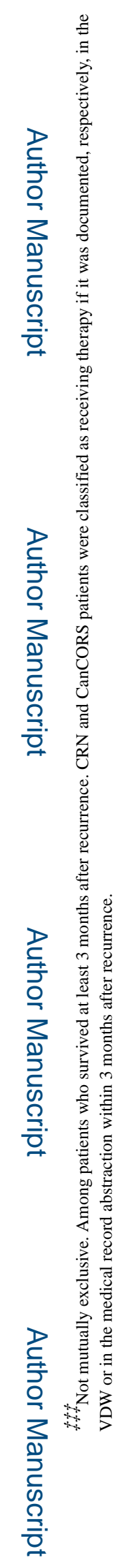




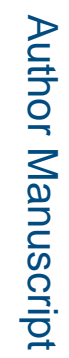

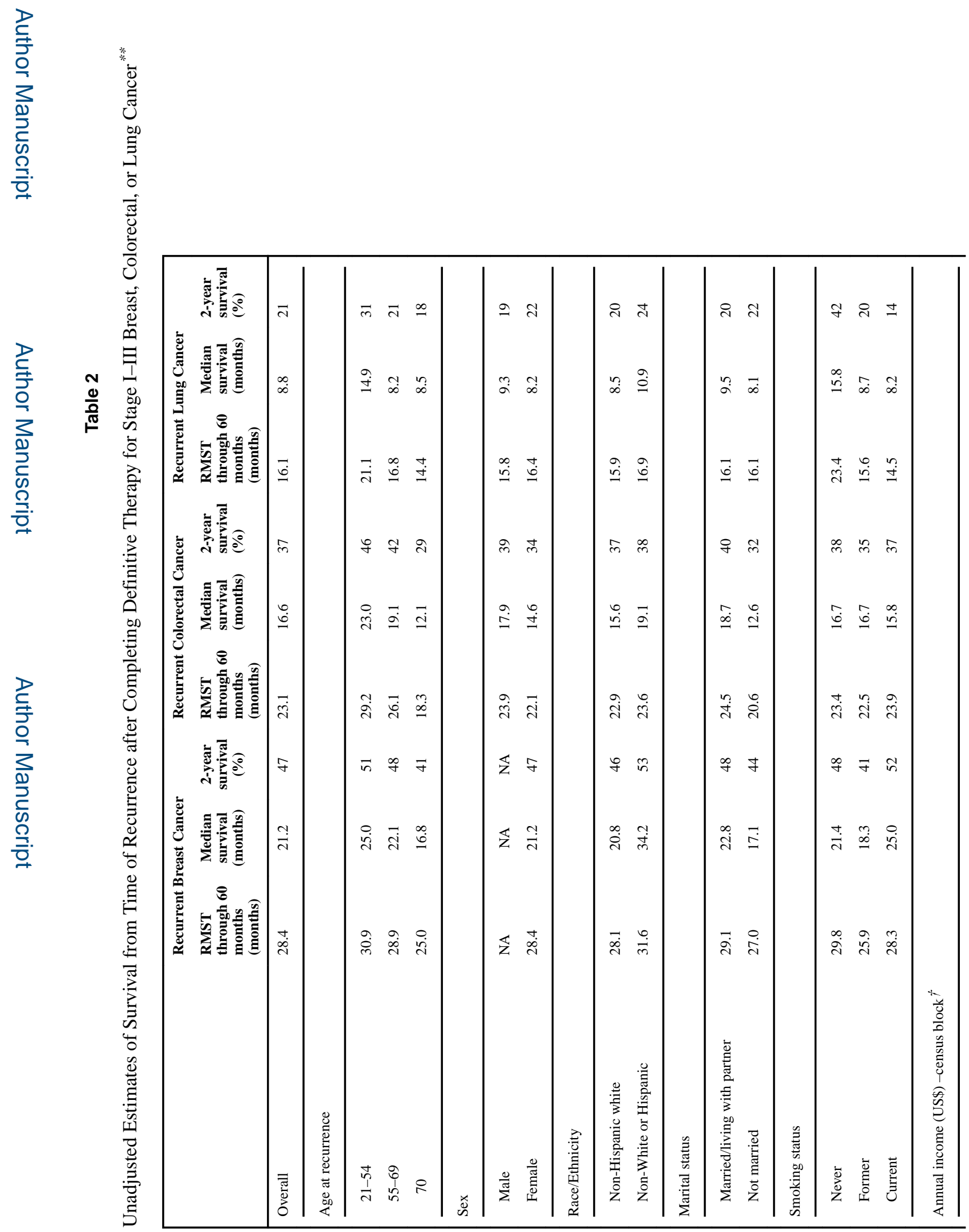




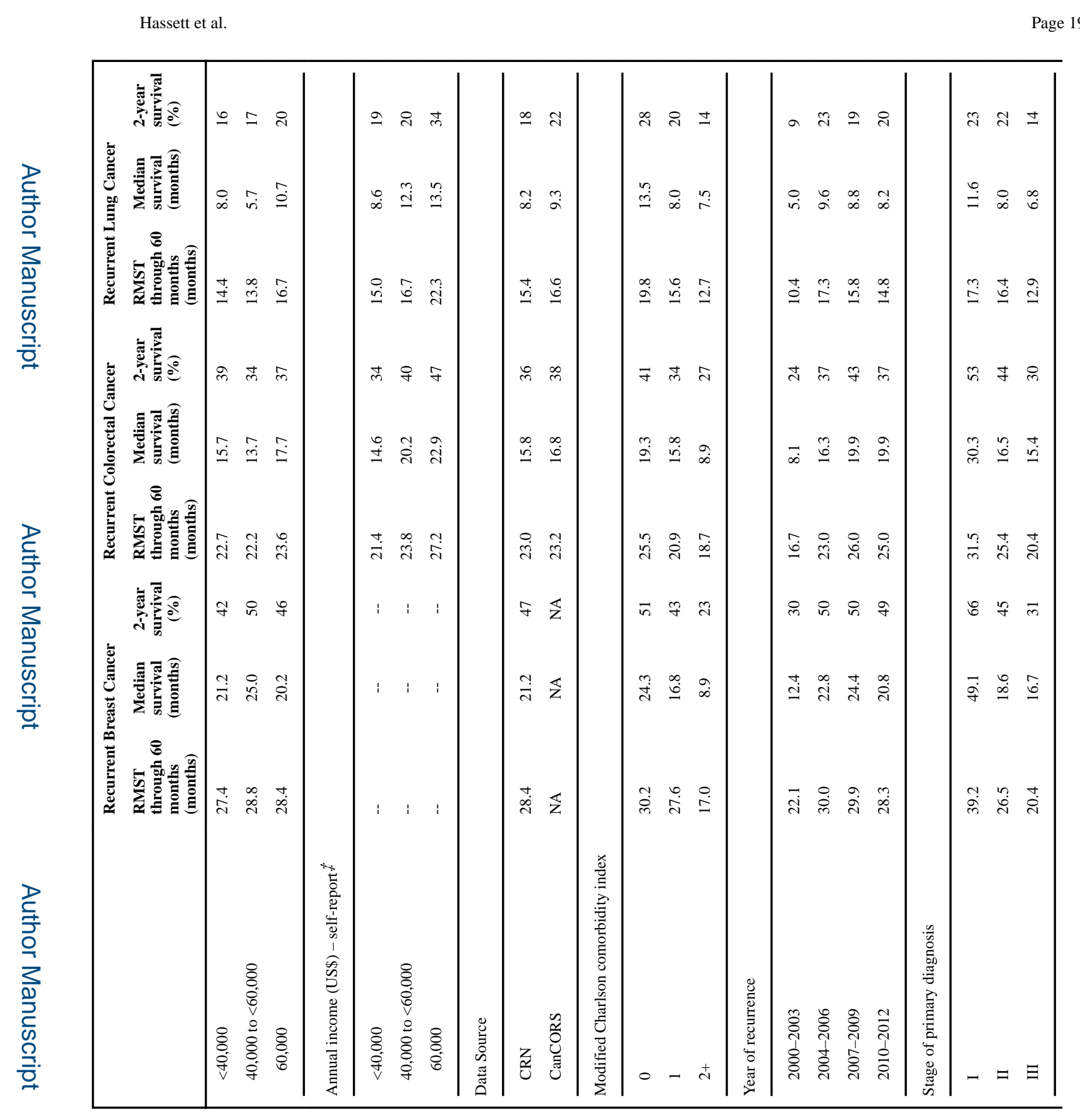

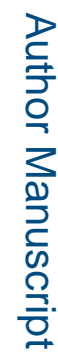




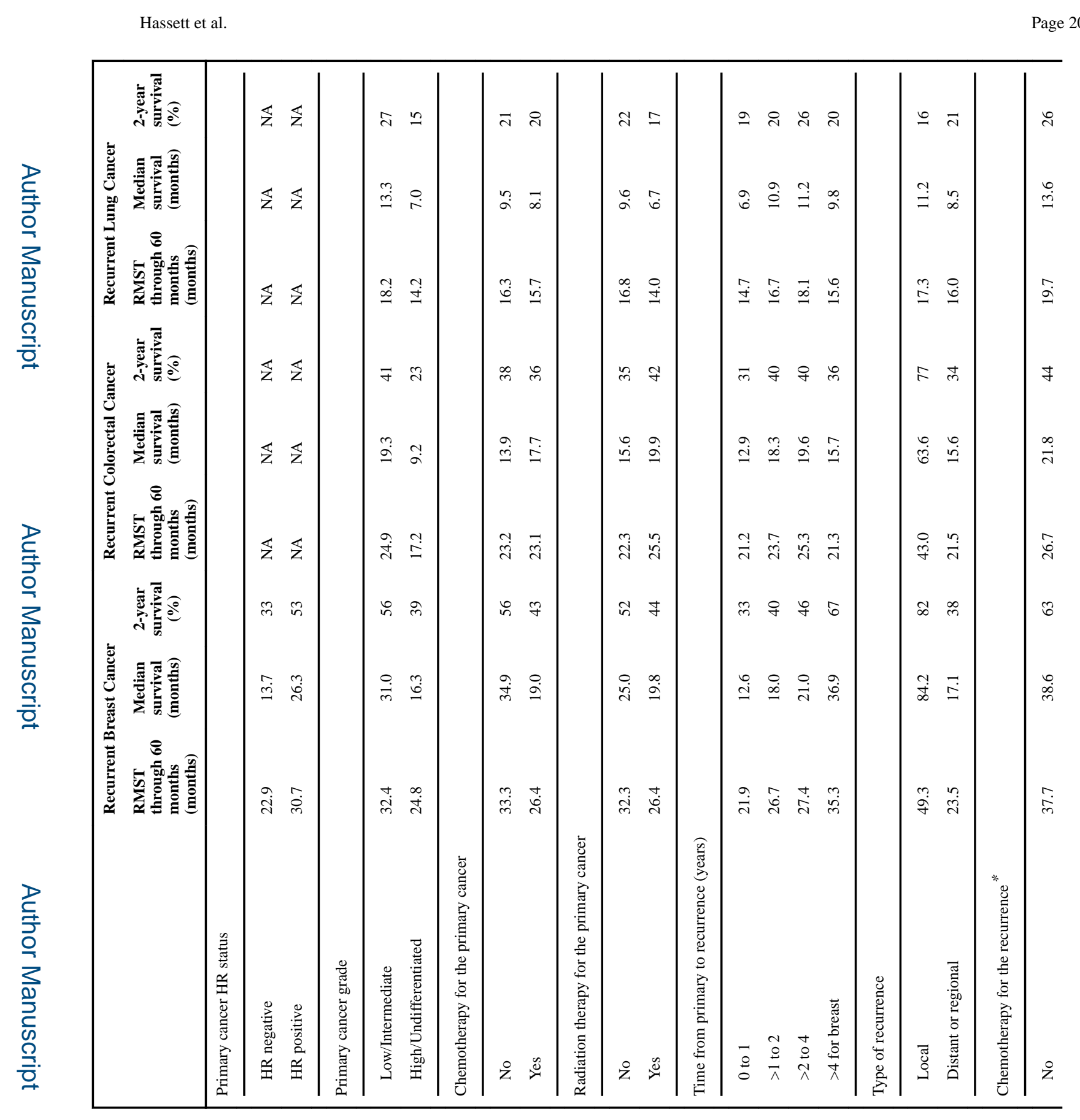

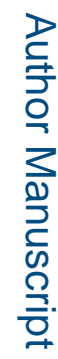




$$
1
$$




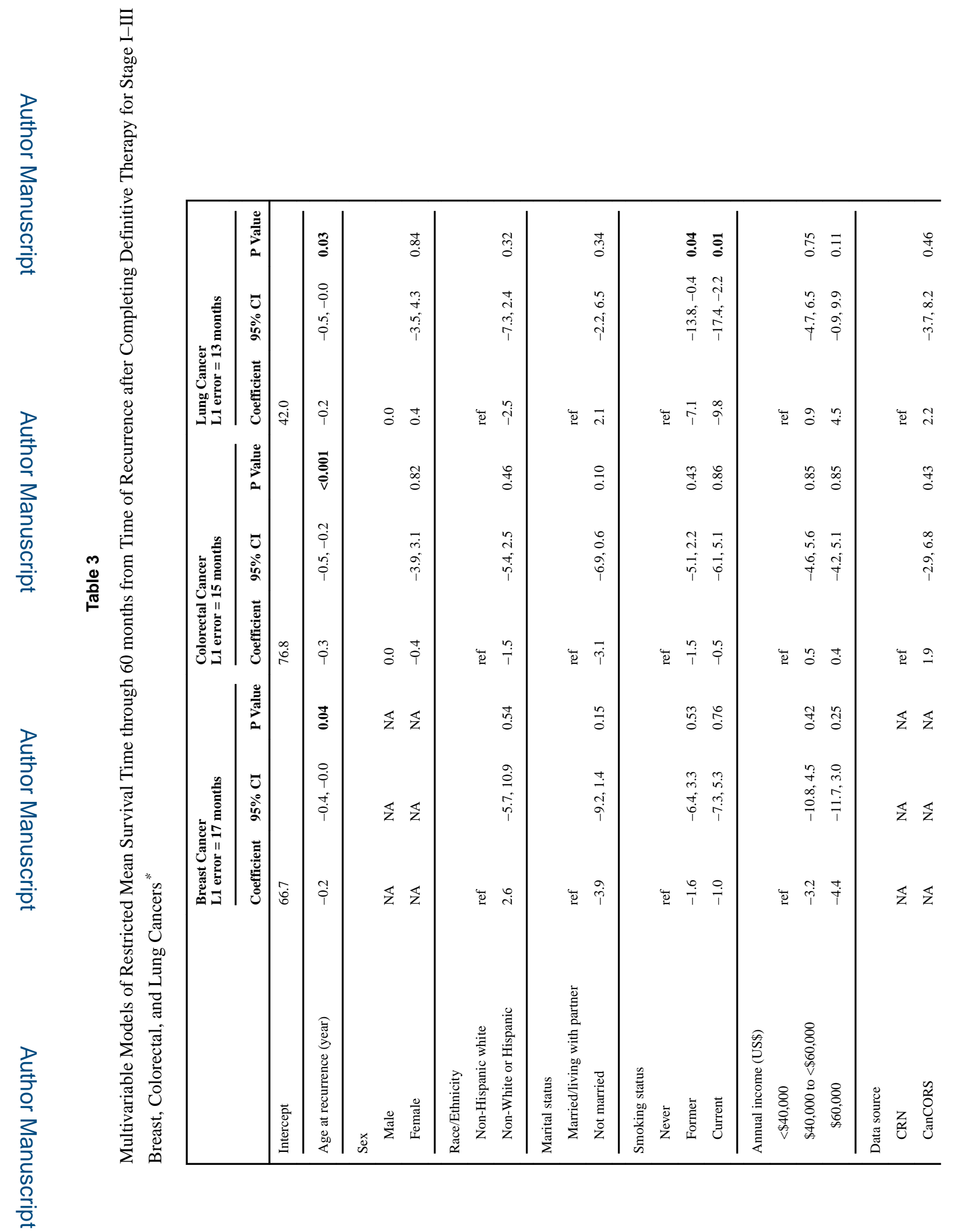

Cancer Epidemiol. Author manuscript; available in PMC 2018 August 01. 


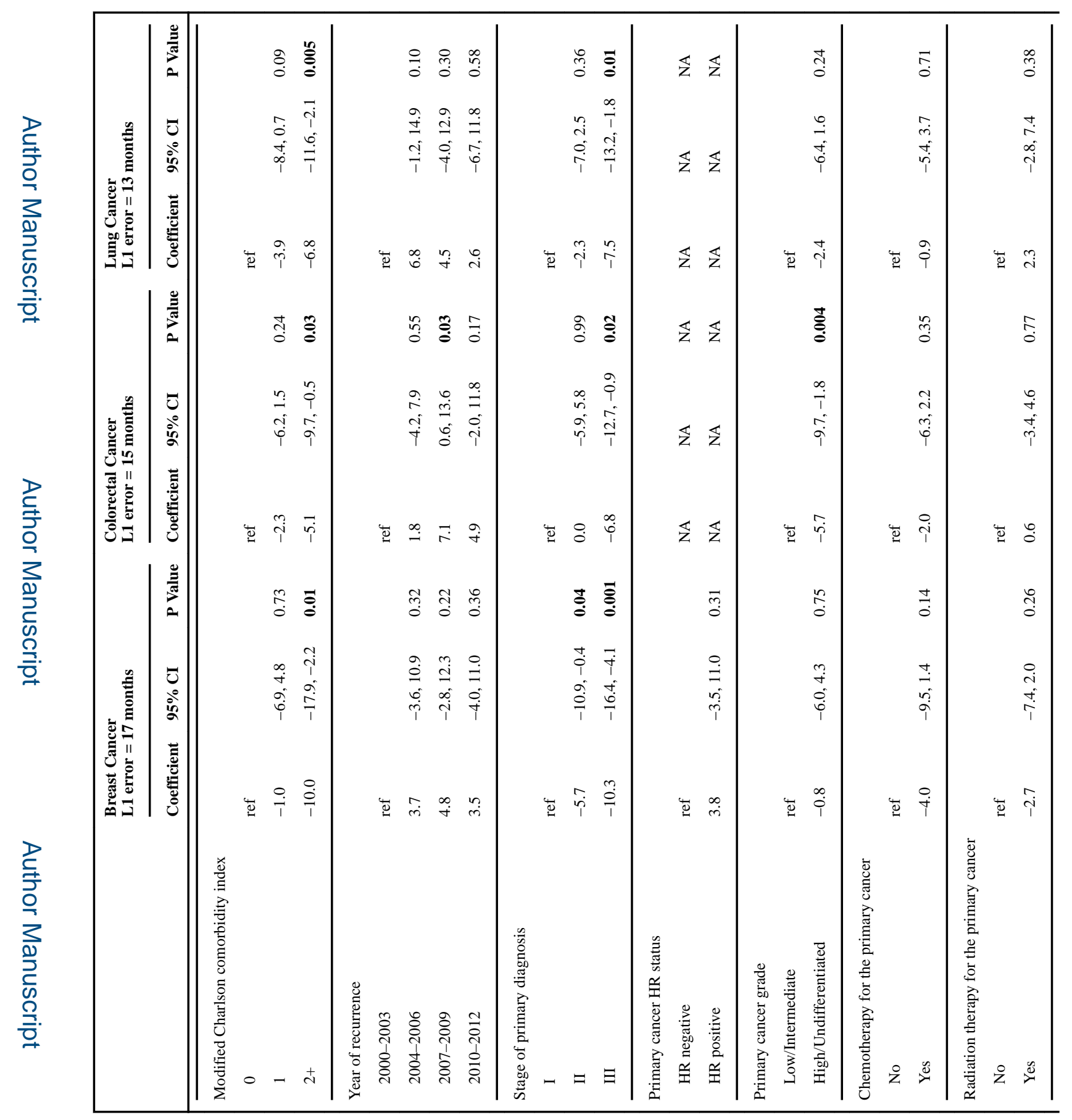

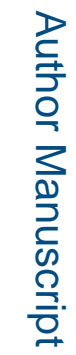




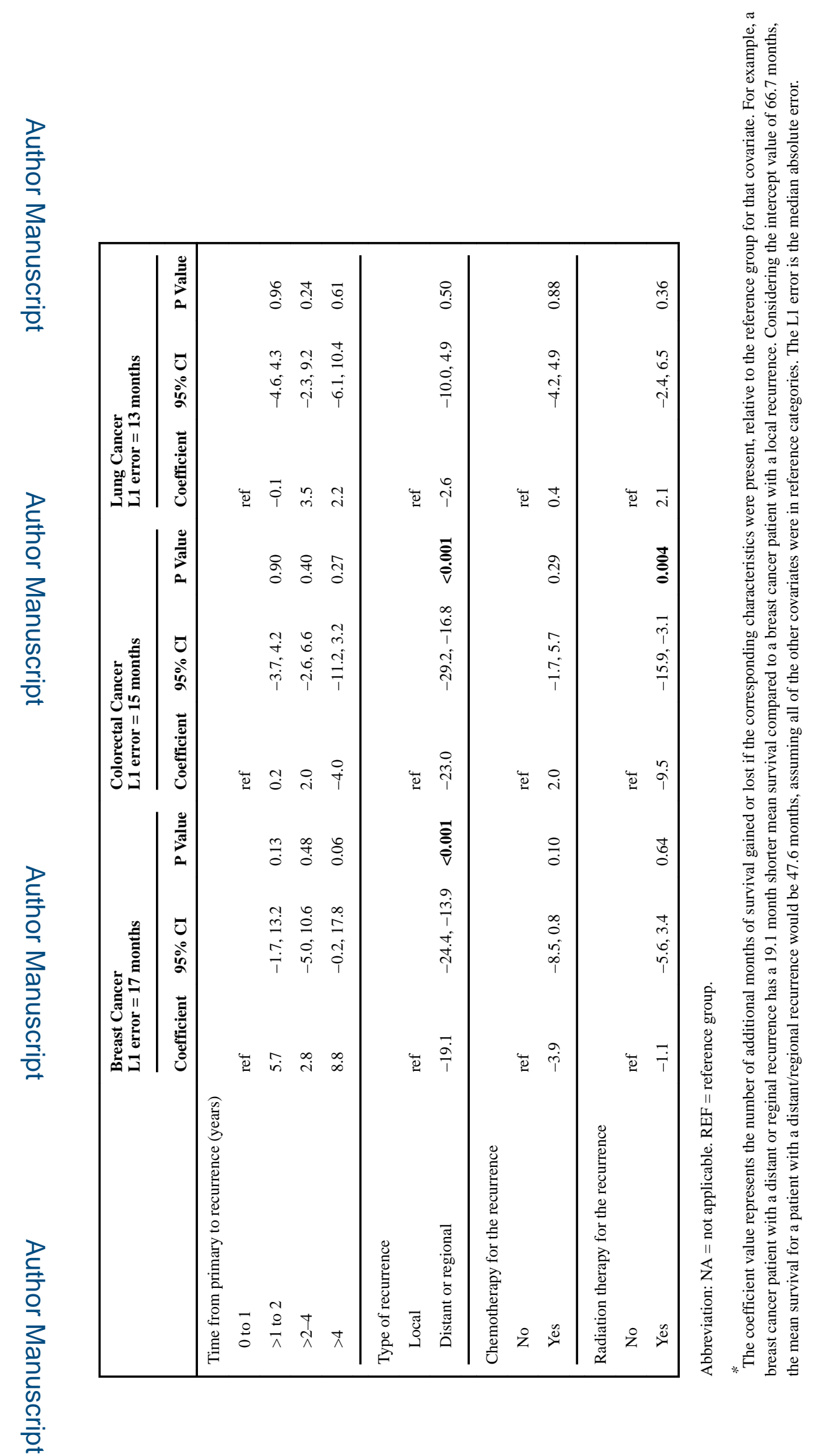

Cancer Epidemiol. Author manuscript; available in PMC 2018 August 01. 